\title{
Reducing the gravitational lensing scatter of type la supernovae without introducing any extra bias
}

\author{
J. Jönsson ${ }^{1}$, E. Mörtsell ${ }^{2}$, and J. Sollerman ${ }^{3,4}$ \\ 1 University of Oxford Astrophysics, Denys Wilkinson Building, Keble Road, Oxford OX1 3RH, UK \\ e-mail: jacke@astro.ox.ac.uk \\ 2 Physics Department, Stockholm University, AlbaNova University Center, 10691 Stockholm, Sweden \\ 3 Stockholm Observatory, AlbaNova, Department of Astronomy, 10691 Stockholm, Sweden \\ ${ }^{4}$ Dark Cosmology Centre, Niels Bohr Institute, University of Copenhagen, Juliane Maries Vej 30, 2100 Copenhagen $\emptyset$, Denmark
}

Received 25 September 2008 / Accepted 14 October 2008

\section{ABSTRACT}

\begin{abstract}
Aims. Magnification and de-magnification due to gravitational lensing will contribute to the brightness scatter of type Ia supernovae ( $\mathrm{SNe}$ Ia). The purpose of this paper is to investigate the possibility of decreasing this scatter by correcting individual SNe Ia using observations of galaxies in the foreground, without introducing any extra bias.

Methods. We simulate a large number of SN Ia lines of sight populated by galaxies. For each line of sight the true magnification factor and an estimate thereof are calculated. The estimated magnification factor corresponds to what an observer would infer from a survey like the SNLS. Using the simulated data we investigate the possibility of estimating the magnification of individual supernovae with enough precision to be able to correct their brightness for gravitational lensing with negligible bias.

Results. Our simulations show that the bias arising from gravitational lensing corrections of individual SNe Ia is negligible for current and next generation surveys and that the scatter from lensing can be reduced by approximately a factor of 2 . The total scatter in the SN Ia magnitudes could be reduced by $4 \%$ for an intrinsic dispersion of 0.13 mag. For an intrinsic dispersion of 0.09 mag, which may be feasible for future surveys, the total scatter could be reduced by $6 \%$. This will reduce the errors on cosmological parameters derived from supernova data by $4-8 \%$. The prospect of correcting for lensing is thus very good.
\end{abstract}

Key words. star: supernovae: general - gravitational lensing - cosmology: large-scale structure of Universe cosmology: cosmological parameters - cosmology: observations

\section{Introduction}

Large dedicated supernova surveys, such as SNLS (Astier et al. 2006), ESSENCE (Miknaitis et al. 2007; Wood-Vasey et al. 2007), and SDSS-II (Frieman et al. 2008), have gathered data on a large number of supernovae at cosmological redshifts. Consequently, supernova cosmology has now reached a stage where systematic uncertainties, rather than lack of statistics, limit the ability to constrain cosmological models. Extinction by dust and peculiar motions of host galaxies seem to be the systematic effects which currently pose the largest difficulties (e.g. Leibundgut 2008). In this paper we investigate the possibility of correcting for another systematic uncertainty - gravitational lensing.

In an inhomogeneous universe like ours, light from a distant type Ia supernova (hereafter SN Ia) is inevitably affected by gravitational lensing. Weak gravitational lensing, which is what we will consider in this paper, refers to the phenomena which occur when a bundle of light rays is distorted by the gravitational fields exerted by matter. The distortion can lead to magnification or de-magnification of the SN Ia flux. This (de)magnification can be described by the magnification factor, $\mu$, which depends on the distribution and composition of the foreground matter. Both baryonic and dark matter influence the trajectories of photons, but since the latter dominates on the scales relevant for this work, we only use the baryonic component as a tracer of the dark matter. If an observer residing in a homogeneous universe would measure the flux $f$, an observer situated in an inhomogeneous universe will measure the flux $f_{\text {obs }}=\mu f$. Due to flux conservation, as long as we do not have multiple images, the probability distribution function for the magnification factor, $P(\mu)$, satisfies the constraint $\langle\mu\rangle=\int P(\mu) \mu \mathrm{d} \mu=1$, i.e. the average magnification factor is unity. The effect of gravitational lensing consequently averages out, which implies that this systematic effect can be controlled by observing large numbers of SNe Ia. In reality however, $\langle\mu\rangle$ converges very slowly towards unity as the sample size increases (Holz \& Linder 2005). This is due to the fact that the whole distribution of $P(\mu)$, including its elusive high magnification tail, must be sampled, which in addition to large numbers also requires large survey areas (Cooray et al. 2006).

Although the bias in principle can be decreased by large numbers, gravitational lensing still contributes to the brightness scatter of SNe Ia. This contribution increases with redshift and is expected to be substantial at $z \gtrsim 1$ (Holz \& Linder 2005). For precision measurements of cosmological parameters it is therefore desirable to minimize this scatter by measuring and correcting for the gravitational lensing effect.

The method we rely upon is based on modeling the dark matter haloes, which surround galaxies, using the observed properties of the galaxies. This method takes into account contributions to the lensing from sub-arc-minute scales, which makes it a viable method, in contrast to shear maps (Dalal et al. 2003) which are insensitive to these scales. The possibility of correcting individual SNe Ia for gravitational lensing using the method considered here was investigated by Gunnarsson et al. (2006), where 
simulations showed that the lensing scatter could be reduced by almost a factor of 3 for a source at redshift $z=1.5$. In this paper we focus on the possibility of correcting for gravitational lensing at the lower redshifts $(z \lesssim 1)$ accessible to the aforementioned large ground based surveys. The simulations aim at mimicking the supernova legacy survey (SNLS, Astier et al. 2006), and follow closely the strategy by Jönsson et al. (2008, hereafter J08).

For a correction to be useful the following two criteria must be met:

1. the correction should decrease the scatter in SN Ia magnitudes due to gravitational lensing;

2. any bias introduced in the SN Ia magnitudes from the correction should be negligible.

In Sect. 2, we use simulated data to investigate the possibility of performing corrections satisfying the two criteria above. The method we use to correct for lensing is explained and applied to the simulated data in Sect. 3. Our results are discussed and summarized in Sect. 4.

\section{Simulations}

Since our simulations are very similar to the ones presented in more detail by $\mathrm{J} 08$, we only provide a brief presentation here.

We have simulated deep galaxy catalogs with properties (galaxy positions, redshifts, luminosities, types) based on real observations (Dahlén et al. 2005). For a single line of sight we first compute the true magnification factor, $\mu_{\text {true }}$, using the multiple lens plane algorithm implemented in the Q-LET package (Gunnarsson 2004). All galaxies are included in the calculation and the dark matter haloes surrounding each galaxy are modeled by truncated Navarro-Frenk-White (NFW, Navarro et al. 1997) profiles. Then we compute the magnification factor which an observer (with access to SNLS data) would estimate, $\mu_{\text {est }}$, for the same line of sight. The observer would, for example, only see galaxies brighter than the magnitude limit $\left(i_{A B}^{\prime} \leq 25.5\right)$ of the survey and would probably have access only to photometric redshifts (with a precision of $\sigma_{\Delta z /(1+z)} \simeq 0.03$ for most galaxies, but failing catastrophically for $2-6 \%$ of the galaxies). The content of the line of sight is therefore rather different when the true and estimated magnifications are calculated. Since the observer does not know what the correct model is, there is also a difference in the model used to compute the true and the estimated magnification factor. Since the effects of gravitational lensing are redshift dependent, we simulate gravitational lensing of sources in the redshift range $0.2 \leq z \leq 1.1$, relevant for e.g. the SNLS survey.

The result of the simulations are hence pairs of true, $\mu_{\text {true }}$, and estimated, $\mu_{\text {est }}$, magnification factors. Since most cosmology fits to date have been performed using magnitudes rather than fluxes, we treat magnification in terms of logarithmic units. Throughout the paper we denote true and estimated magnifications by $\Delta m_{\text {true }}=-2.5 \log _{10} \mu_{\text {true }}$ and $\Delta m_{\text {est }}=-2.5 \log _{10} \mu_{\text {est }}$, respectively.

Figure 1 shows simulated pairs of magnification factors in logarithmic units. This plot shows most of the simulated pairs, but not the small fraction $(\lesssim 0.3 \%)$ belonging to the high magnification tail with $\Delta m_{\text {true }} \lesssim-0.3$. The scatter in this plot comes mostly from the uncertainty in the model used to convert galaxy luminosity to mass (in our case Tully-Fisher and FaberJackson relations). Given the importance of the luminosity-tomass model, we continue this investigation by considering three different scenarios, all shown in Fig. 1:

1. no shift in halo masses (circles);

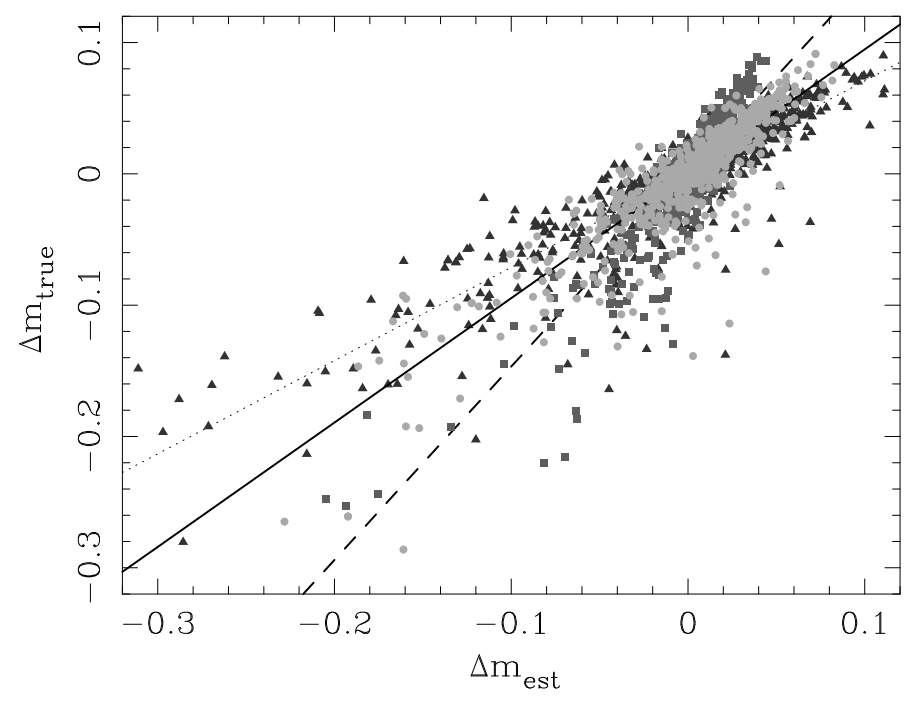

Fig. 1. True magnification, $\Delta m_{\text {true }}$, versus estimated magnification, $\Delta m_{\mathrm{est}}$, for three different scenarios: no shift in halo masses (circles), underestimated halo masses (squares), and overestimated halo masses (triangles). The straight lines show the best fits to the no shift (solid line), underestimation (dashed line), and overestimation (dotted line) scenario.

2. underestimated halo masses (squares);

3. overestimated halo masses (triangles).

If the masses of the dark matter haloes are substantially overestimated (by $50 \%$ in scenario 2) or underestimated (by $50 \%$ in scenario 3), the gravitational magnification estimates will be erroneous. Our simulations aim to investigate to what extent a proper correction can be made for the lensing magnification given these uncertainties. Other sources of uncertainty, such as scatter in the luminosity-to-mass model, were also included for all three scenarios. Clearly, the distributions of points in the plot differ for the three scenarios. True and estimated magnification factors are apparently correlated in all scenarios, albeit with different slopes. The no shift scenario has a slope (outlined by the solid line in Fig. 1) near unity, which reflects the fact that the model used to estimate the magnification is very close to the correct one. The underestimation (dashed line) and overestimation (dotted line) scenarios have slopes which differ from unity.

From Fig. 1, the asymmetry of both the distribution of $\Delta m_{\text {true }}$ and $\Delta m_{\mathrm{est}}$ is evident. For slopes differing from unity this asymmetry implies an asymmetry in the errors in the estimated magnifications. The estimated magnification of a SN Ia in the high magnification tail (negative values in Fig. 1) is likely to be more erroneous than a typical SN Ia which is slightly de-magnified (positive values in Fig. 1).

\section{Gravitational lensing corrections}

\subsection{The magnification-residual diagram}

Since gravitationally magnified (de-magnified) SNe Ia should be brighter (fainter) than the average supernova, a correlation between estimated magnification and SN Ia brightness is expected. According to Fig. 1, which can be seen as an idealized magnification-residual diagram, a correlation is expected even if the model used to estimate the magnification factors is biased. A real magnification-residual diagram, where Hubble diagram residuals are used instead of $\Delta m_{\text {true }}$, will also be smeared by, e.g., measurement errors and intrinsic brightness scatter. We 




Fig. 2. Bias (panel a)) and dispersion (panel b)) in $\epsilon$ as a function of redshift. The no shift scenario is represented by the thin solid curves. Dashed and dotted curves correspond to the under- and overestimation scenarios, respectively. Bias and dispersion of $\Delta m_{\text {true }}$, outlined by the thick solid curves, are also shown for comparison. The estimated magnifications, $\Delta m_{\mathrm{est}}$, were used to correct for gravitational lensing.

take the supernova Hubble diagram residuals to be the differences between observed magnitudes, $m_{\mathrm{obs}}$, and magnitudes predicted by a cosmological model of a homogeneous universe (we assume a flat universe, dominated by a cosmological constant, with $\Omega_{\mathrm{M}}=0.3$ and $H_{0}=70 \mathrm{~km} \mathrm{~s}^{-1} \mathrm{Mpc}^{-1}$ ). In the following, we assume that the residuals have been computed using the correct cosmological model. The residual is then the sum of $\Delta m_{\text {true }}$ and noise, $\Delta m_{\text {noise }}$, from, e.g., intrinsic SN Ia brightness scatter and measurement errors. We ignore other systematic sources of uncertainty and assume the noise to be Gaussian.

\subsection{Magnitude bias}

Because of gravitational lensing, an observer in an inhomogeneous universe measures the magnitude

$m_{\mathrm{obs}}=m+\Delta m_{\mathrm{true}}$

rather than the magnitude $m=-2.5 \log _{10} f$, which would be measured if the observer resided in a homogeneous universe. The effect of gravitational lensing averages out because the average magnification factor is unity. However, an infinite number of $\mathrm{SNe}$ Ia is in principle required, since the average magnification factor of a finite sample converges very slowly to unity as the sample size increases (see Fig. 2 in Holz \& Linder 2005). When magnitudes are used instead of fluxes, on the other hand, the effect no longer averages out even in principle, because $\left\langle\log _{10} \mu\right\rangle \neq$ 0 even though $\langle\mu\rangle=1$. Observed magnitudes are consequently biased by an amount depending on the redshift. The thick solid curve in Fig. 2a shows this bias, $\left\langle m_{\text {obs }}\right\rangle-m=\left\langle\Delta m_{\text {true }}\right\rangle$, which is of the order $10^{-3}$.

Recently Sarkar et al. (2008) investigated the expected bias in the dark energy equation of state parameter due to gravitational lensing for future SN Ia surveys. In their study Sarkar et al. (2008) considered both averaging over magnitudes (as we do here) and a flux-averaging technique (Wang 2000; Wang \& Mukherjee 2004). In both cases they found the resulting bias in the equation of state parameter to be negligible, compared to the expected statistical uncertainty.

\subsection{Lensing corrections}

If the estimated magnification factors are used to correct for gravitational lensing, the corrected magnitudes would be

$m_{\mathrm{corr}}=m_{\mathrm{obs}}-\Delta m_{\mathrm{est}}=m+\epsilon$,

where the correction is characterized by the difference

$\epsilon=\Delta m_{\text {true }}-\Delta m_{\mathrm{est}}$.

For the correction to be useful, according to the first criterion stated above, the dispersion in the SN Ia magnitudes must be decreased $\left(\sigma_{m_{\text {corr }}}<\sigma_{m_{\text {obs }}}\right)$, which consequently means that the dispersion in $\epsilon$ must be smaller than the dispersion in $\Delta m_{\text {true }}$, i.e. $\sigma_{\epsilon} / \sigma_{\Delta m_{\text {true }}}<1$.

The second criterion requires that the correction does not introduce any extra bias in the corrected magnitudes. According to Eq. (2) this bias is given by $\langle\epsilon\rangle$. Whether the bias is negligible or not depends on the size of the statistical error. We require $\langle\epsilon\rangle / \sigma_{\Delta m_{\text {true }}}<1$ for a correction to be considered useful.

Figures $2 \mathrm{a}$ and $\mathrm{b}$ show the bias and dispersion, respectively, for $\epsilon$ computed for the three different scenarios discussed in Sect. 2 over a range of redshifts. In this case, no noise has been taken into account. The scenario corresponding to no shift in halo mass is shown by the thin solid curves. Thin dashed and dotted curves outline the results for the scenarios were halo masses have been under- and overestimated, respectively. We also display with the thick solid curves the bias and dispersion in $\Delta m_{\text {true }}$. For all three scenarios, the first criterion above is fulfilled, i.e. $\sigma_{\epsilon}<\sigma_{\Delta m_{\text {true }}}$ at all redshifts. At $z \simeq 0.8$, which is the redshift where the SNLS distribution of SNe Ia peaks, the reduction in the scatter is a factor $2.1,1.9$, and 1.8 for scenario 1,2 , and 3 . The absolute value of the bias, $|\langle\epsilon\rangle|$, is $\$ 3 \times 10^{-3}$ for all scenarios. If this bias is divided by $\sigma_{\Delta m_{\text {true }}}$, we find $\left|\langle\epsilon\rangle / \sigma_{\Delta m_{\text {true }}}\right| \lesssim 4 \times 10^{-2}$, which implies successful corrections for all cases and at all redshifts according to our second criterion. Moreover, for all three scenarios $|\langle\epsilon\rangle|\left\langle\left|\left\langle\Delta m_{\text {true }}\right\rangle\right|\right.$. We have thereby demonstrated that such a correction for gravitational lensing would clearly decrease the scatter in the Hubble diagram without adding significant bias after the corrections.

Let us now investigate the potential benefits of gravitational lensing corrections in the presence of realistic intrinsic brightness scatter and measurement errors. We have simulated SNLSlike data sets, consisting of $250 \mathrm{SNe}$ Ia each, for the three scenarios. Since the results depend on the assumed errors, we have also varied the intrinsic brightness scatter in the simulations. To model the measurement errors we have used the following fit to the first year SNLS data (Astier et al. 2006):

$\sigma_{\text {err }}= \begin{cases}0.05 \mathrm{mag} & \text { if } z<0.8 \\ 0.84 z^{2}-1.04 z+0.34 \mathrm{mag} & \text { if } z \geq 0.8\end{cases}$

For each simulated data set, $\sigma_{\Delta m_{\text {true }}}, \sigma_{\epsilon}$, and $\langle\epsilon\rangle$ were computed. Figure 3 shows scatter plots of $\langle\epsilon\rangle / \sigma_{\Delta m_{\text {true }}}$ (the second criterion) versus $\sigma_{\epsilon} / \sigma_{\Delta m_{\text {true }}}$ (the first criterion) for different assumed errors. As in Fig. 1 the three scenarios are indicated by different plotting symbols. In Fig. 3 a measurement errors, modeled by Eq. (4), and intrinsic dispersion, $\sigma_{\text {int }}=0.13 \mathrm{mag}$, correspond to the precision of first year SNLS data. A future decrease in the intrinsic dispersion is possible. We therefore show in Fig. $3 b$ results obtained for the same measurement errors, but with $\sigma_{\text {int }}=0.09$ mag. Figure $3 \mathrm{c}$ shows results for $\sigma_{\text {int }}=0.09 \mathrm{mag}$ and negligible measurement errors, i.e. $\sigma_{\text {err }}=0$ mag. For comparison we also show results in Fig. $3 \mathrm{~d}$ for an intrinsic dispersion of only 0.05 mag and $\sigma_{\text {err }}=0 \mathrm{mag}$. The distributions of points in the scatter plots are rather similar irrespective of the scenario. A value 


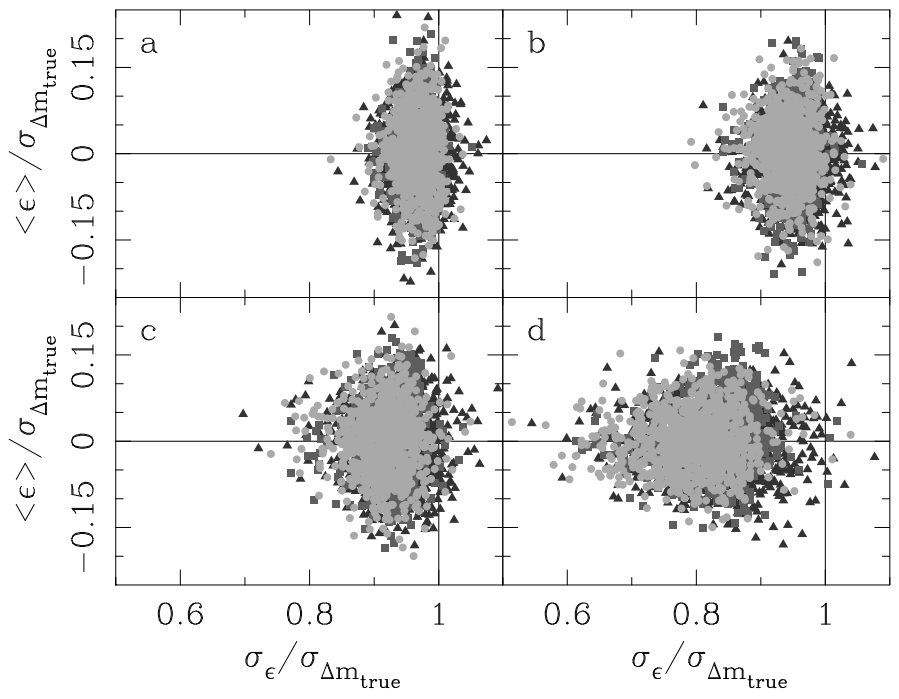

Fig. 3. Scatter plot of $\langle\epsilon\rangle / \sigma_{\Delta m_{\text {true }}}$ versus $\sigma_{\epsilon} / \sigma_{\Delta m_{\text {true }}}$ for simulated SNLSlike data sets consisting of $250 \mathrm{SNe}$ Ia each. Circles, squares, and triangles represent the no shift, under-, and overestimation scenarios, respectively. The different panels correspond to different intrinsic brightness dispersion and measurement errors: panel a) $\sigma_{\text {int }}=0.13 \mathrm{mag}, \sigma_{\text {err }}$ given by Eq. (4); panel b) $\sigma_{\text {int }}=0.09 \mathrm{mag}, \sigma_{\text {err }}$ given by Eq. (4); panel c) $\sigma_{\text {int }}=0.09 \mathrm{mag}, \sigma_{\text {err }}=0 \mathrm{mag}$; panel d) $\sigma_{\text {int }}=0.05 \mathrm{mag}, \sigma_{\text {err }}=0$ mag.

of $\sigma_{\epsilon} / \sigma_{\Delta m_{\text {true }}}$ less than unity, i.e. to the left of the vertical lines, indicates a successful correction according to the first criterion. The number of simulated data sets failing to meet the first criterion, i.e. points to the right of the vertical line, decreases as the errors decrease. In Fig. 3a the correction fails for 4\%, 1\%, and $10 \%$ of the synthetic data sets for the no shift, underestimation, and overestimation scenario. These numbers drop to less than one percent for Fig. 3d.

From Fig. 3 it is also evident that the bias after the correction has been performed is small; for all cases $\left|\langle\epsilon\rangle / \sigma_{\Delta m_{\text {true }}}\right|<0.25$. According to Fig. 3, corrections for gravitational lensing are likely to reduce the scatter in SN Ia brightness without introducing any harmful bias.

Figure 4 shows a projection of Fig. 3 focusing on the distribution of the ratio $\sigma_{\epsilon} / \sigma_{\Delta m_{\text {true }}}$. Figures $4 \mathrm{a}-\mathrm{c}$ show results obtained for the no shift, under-, and overestimation scenarios. Solid and dashed curves correspond to measurement errors modeled by Eq. (4) and an intrinsic dispersion of 0.13 and 0.09 mag, respectively. Dotted and dash-dotted curves correspond to negligible measurement errors ( $\left.\sigma_{\text {err }}=0 \mathrm{mag}\right)$ and intrinsic dispersions of 0.09 and $0.05 \mathrm{mag}$, respectively. In Table 1, the characteristics of the distributions shown in Fig. 4 are summarized. From Fig. 4, it is clear that the distributions obtained with systematic shifts in the halo masses (scenario 2 and 3 ) are similar to the distributions corresponding to the no shift scenario. From Table 1 we see that $0.96 \leq\left\langle\sigma_{\epsilon} / \sigma_{\Delta m_{\text {true }}}\right\rangle \leq 0.97$ when the intrinsic scatter is $\sigma_{\text {int }}=0.13 \mathrm{mag}$, which implies that the average effect of correcting for gravitational lensing is to decrease the total scatter by $3-4 \%$. A realistic future intrinsic dispersion of $\sigma_{\text {int }}=0.09$ mag would allow a decrease in the total scatter by $5-6 \%$, for measurement errors modeled by Eq. (4), and by 7-8\% if measurement errors could be reduced to a negligible level.

We have also investigated the possible improvements on cosmological parameter estimation that could be gained from gravitational lensing corrections. Using the Fisher information matrix, we computed confidence ellipses in the $\left(\Omega_{\mathrm{M}}, w\right)$-plane assuming a flat universe. The parameters $\Omega_{\mathrm{M}}$ and $w$ denote the

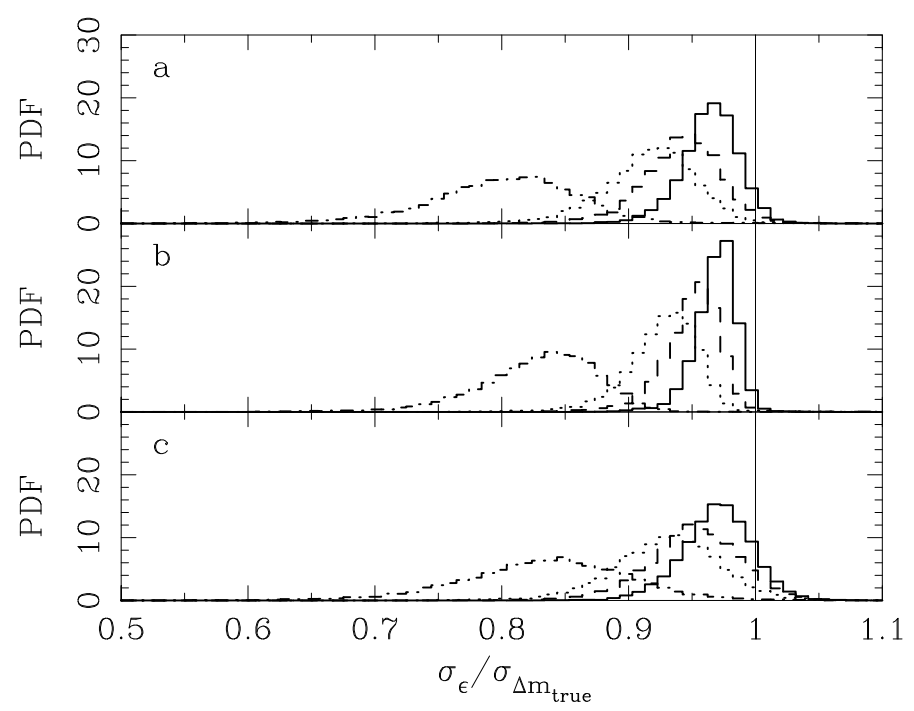

Fig. 4. Probability distribution functions of $\sigma_{\epsilon} / \sigma_{\Delta m_{\text {true }}}$ for different scenarios and errors. The distributions were obtained for simulated SNLSlike data sets consisting of $250 \mathrm{SNe}$ Ia. Panels a), b), and c) correspond to the no shift, under-, and overestimation scenario. The curves show results for different assumed errors: solid curves correspond to $\sigma_{\text {err }}$ given by Eq. (4) and $\sigma_{\text {int }}=0.13 \mathrm{mag}$; dashed curves correspond to $\sigma_{\text {err }}$ given by Eq. (4) and $\sigma_{\text {int }}=0.09 \mathrm{mag}$; dotted curves correspond to $\sigma_{\text {err }}=0$ and $\sigma_{\text {int }}=0.09 \mathrm{mag}$; dash-dotted curves correspond to $\sigma_{\text {err }}=0$ and $\sigma_{\text {int }}=0.05$ mag.

dimensionless matter density of the universe and a constant dark energy equation of state parameter, respectively. In this analysis, a low and a high redshift SN Ia data set were used. The high redshift data set consists of $250 \mathrm{SNe}$ Ia with redshifts and measurements errors similar to first year SNLS data (Astier et al. 2006). The purpose of the low redshift data set, consisting of 44 SNe Ia unaffected by lensing, is to anchor the Hubble diagram. Redshifts and uncertainties were similar to the low redshift data set used in Astier et al. (2006). Errors due to gravitational lensing before and after correction were modeled using the curves in Fig. 2. Our treatment of the errors is hence simplified, because these curves correspond to the dispersion and do not take the asymmetry of the distributions into account. Table 2 shows the relative improvement in the area of the confidence level ellipses due to gravitational lensing corrections, $A_{\text {corr }} / A_{\text {lensed }}$. The scatter due to gravitational lensing is rather small compared to intrinsic brightness dispersion and measurement errors. Nevertheless, corrections for gravitational lensing would result in improvements of 4-8\% for realistic values of the intrinsic dispersion and $\sim 30 \%$ for a very optimistic scenario where other sources of error are negligible and the supernovae are calibrated to 5\% accuracy.

\subsection{Correction coefficient}

In the next step, we could try to improve the correction by considering a model that incorporates the different slopes exhibited in Fig. 1. Since the points in Fig. 1 appear to cluster around straight lines with different slopes, a linear correction in $\Delta m_{\mathrm{est}}$,

$\Delta m_{\mathrm{est}}^{B}=B \Delta m_{\mathrm{est}}$

is the simplest approach. The $B$-coefficient is related to how bad we are at estimating the luminosity-to-mass relation, and correcting with $B$ is the most straightforward way to remedy this. In J08 it was shown that since $B$ is sensitive to the normalization 
Table 1. Characteristics (mean and root mean square) of the distribution of $\sigma_{\epsilon} / \sigma_{\Delta m_{\text {true }}}$ for the no shift, under- and overestimation scenarios and different assumed errors. The distributions were obtained from 10000 simulations of SNLS-like data sets consisting of 250 SNe Ia each.

\begin{tabular}{llllllll}
\hline \hline$\sigma_{\text {int }}$ & \multicolumn{2}{c}{$\sigma_{\text {err }}$} & \multicolumn{2}{c}{ Scenario 1 } & \multicolumn{2}{c}{ Scenario 2 $^{b}$} & \multicolumn{2}{c}{ Scenario 3 } \\
$(\mathrm{mag})$ & $(\mathrm{mag})$ & Mean & RMS & Mean & RMS & Mean & RMS \\
\hline 0.13 & Eq. (4) & 0.96 & 0.02 & 0.97 & 0.02 & 0.97 & 0.03 \\
0.09 & Eq. (4) & 0.94 & 0.03 & 0.95 & 0.02 & 0.95 & 0.04 \\
0.09 & 0 & 0.92 & 0.04 & 0.93 & 0.03 & 0.93 & 0.04 \\
0.05 & 0 & 0.80 & 0.06 & 0.83 & 0.05 & 0.83 & 0.07 \\
\hline
\end{tabular}

a No shift in halo mass.

${ }^{b}$ All halo masses are underestimated by $50 \%$.

${ }^{c}$ All halo masses are overestimated by $50 \%$.

Table 2. Relative improvement in confidence level contours in the $\left(\Omega_{\mathrm{M}}, w\right)$-plane from corrections for gravitational lensing. The results presented in this table were obtained using a Fisher matrix analysis for an SNLS-like data set consisting of 250 high redshift SNe Ia and 44 low redshift $\mathrm{SNe}$ Ia (assumed not to be affected by lensing).

\begin{tabular}{lllll}
\hline \hline $\begin{array}{l}\sigma_{\text {int }} \\
(\mathrm{mag})\end{array}$ & $\begin{array}{l}\sigma_{\text {err }} \\
(\mathrm{mag})\end{array}$ & $\begin{array}{l}\text { Scenario 1 }^{a} \\
A_{\text {corr }} / A_{\text {lensed }}\end{array}$ & $\begin{array}{l}\text { Scenario } 2^{b} \\
A_{\text {corr }} / A_{\text {lensed }}\end{array}$ & $\begin{array}{l}\text { Scenario 3 } \\
A_{\text {corr }} / A_{\text {lensed }}\end{array}$ \\
\hline 0.13 & Eq. (4) & 0.95 & 0.95 & 0.96 \\
0.09 & Eq. (4) & 0.92 & 0.93 & 0.93 \\
0.09 & 0 & 0.84 & 0.87 & 0.87 \\
0.05 & 0 & 0.69 & 0.74 & 0.74 \\
\hline
\end{tabular}

a No shift in halo mass.

${ }^{b}$ All halo masses are underestimated by $50 \%$.

$c$ All halo masses are overestimated by $50 \%$.

of halo masses, we can constrain galaxy masses using the fitted value of $B$.

Since the effects of gravitational lensing are redshift dependent, the correction coefficient could change with redshift. Figure 5 shows the best fit values of $B$ to a large number of simulated pairs of $\Delta m_{\mathrm{est}}$ and $\Delta m_{\text {true }}$ for our three scenarios as a function of redshift. An optimal correction would, of course, use values of $B$ obtained at different redshifts, but would be difficult to derive for a limited data set. Fortunately, the correction coefficient $B$ stays rather constant with redshift, which implies that a slope fitted to a data set spanning a range of redshifts should be useful.

Figure 6, which is is analogous to Fig. 2, shows dispersion and bias as a function of redshift after corrections have been performed using the best fitting correction coefficient, $B$, to the entire data set, which spans a range of redshifts. Equation (5) cannot remove the bias at all redshifts, but can bring the bias for the under- and overestimation scenario down by $\sim 25 \%$ and $\sim 70 \%$, respectively.

Including the $B$-coefficient in the corrections has a negligible impact on $\langle\epsilon\rangle / \sigma_{\Delta m_{\text {true }}}$ for our simulated data sets (see Sect. 3.3), but make a difference to the distributions of $\sigma_{\epsilon} / \sigma_{\Delta m_{\text {est }}}$. Figure 7, which is similar to Fig. 4, shows the distributions for the no shift scenario when corrections have been performed using Eq. (5). Only the distribution corresponding to scenario 1 is shown, because the distributions belonging to the three different scenarios are indistinguishable. Equation (5) thus eliminates the effect of the biased halo masses. Furthermore, all distributions are pushed to the left of the vertical line $\left(\sigma_{\epsilon} / \sigma_{\Delta m_{\text {est }}}=1\right)$. The inclusion of the correction coefficient $B$ thus reduces the corrected

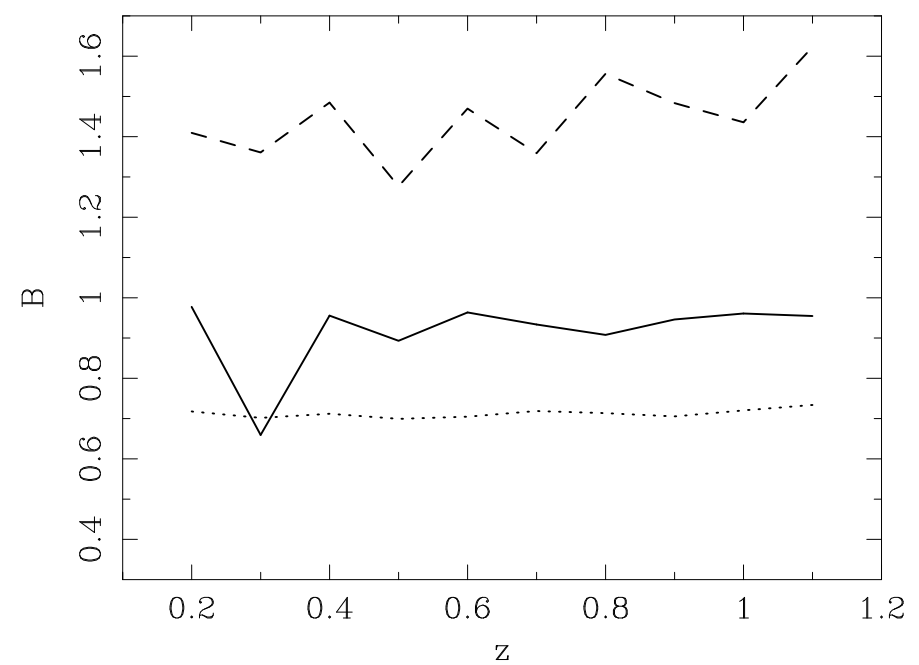

Fig. 5. Correction coefficient $B$ as a function of redshift for three simulated scenarios. For the first scenario (solid curve) there is no systematic shift in halo masses. For the second (dashed curve) and third (dotted curve) scenario halo masses were systematically under- and overestimated by $50 \%$.

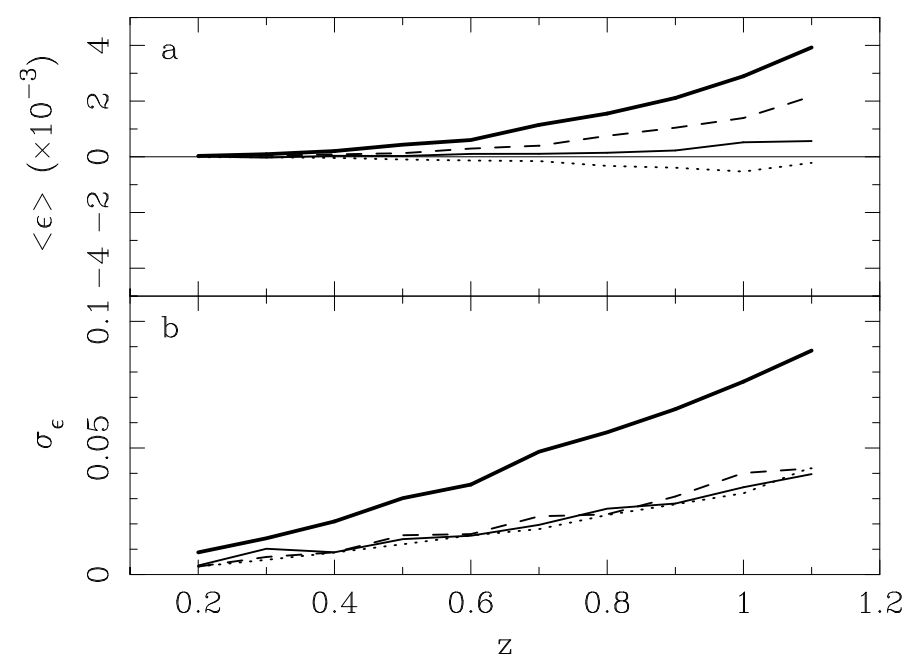

Fig. 6. Bias (panel a)) and dispersion (panel b)) in $\epsilon$ as a function of redshift. The no shift scenario is represented by the thin solid curves. Dashed and dotted curves correspond to the under- and overestimation scenarios, respectively. Bias and dispersion of $\Delta m_{\text {true }}$, outlined by the thick solid curves, are also shown for comparison. To correct for gravitational lensing the formula $\Delta m_{\text {est }}^{B}=B \Delta m_{\text {est }}$ with $B$ corresponding to the value outlined by the straight lines in Fig. 1 was used.

gravitational lensing dispersion down to the level of scenario 1, even if the halo masses are originally over- or underestimated.

It also improves cosmological constraints in the $\left(\Omega_{\mathrm{M}}, w\right)$ plane to the same level as for the no shift scenario without the correction coefficient (see Table 2). No further improvement in the cosmological constraints can be achieved by Eq. (5) for the no shift scenario.

\subsection{Judging the correction}

Correcting for gravitational lensing can of course only be justified if there is a correlation between residuals and estimated magnification. The presence or absence of a correlation after the correction has been performed can be used to judge the success of the correction. If the correction was successful, there should 


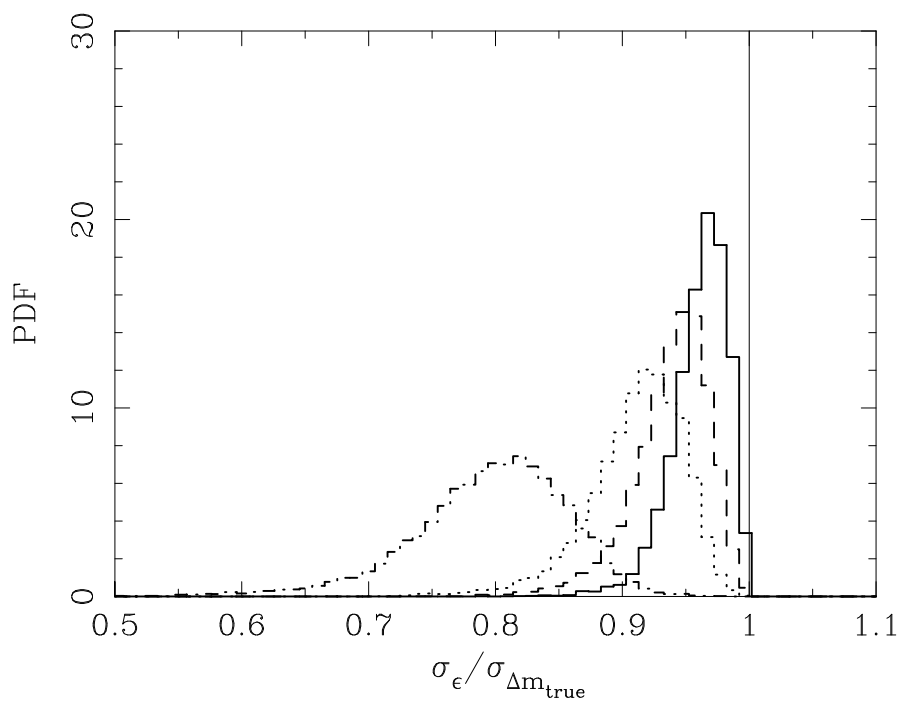

Fig. 7. Probability distribution functions of $\sigma_{\epsilon} / \sigma_{\Delta m_{\text {true }}}$ for the no shift scenario and different errors. The distributions were obtained for simulated SNLS-like data sets consisting of $250 \mathrm{SNe}$ Ia corrected for gravitational lensing using the formula $\Delta m_{\mathrm{est}}^{B}=B \Delta m_{\mathrm{est}}$. The curves show results for different assumed errors: solid curves correspond to $\sigma_{\text {err }}$ given by Eq. (4) and $\sigma_{\text {int }}=0.13 \mathrm{mag}$; dashed curves correspond to $\sigma_{\text {err }}$ given by Eq. (4) and $\sigma_{\text {int }}=0.09 \mathrm{mag}$; dotted curves correspond to $\sigma_{\text {err }}=0$ and $\sigma_{\text {int }}=0.09 \mathrm{mag}$; dash-dotted curves correspond to $\sigma_{\text {err }}=0$ and $\sigma_{\text {int }}=0.05 \mathrm{mag}$.

no longer be any correlation. On the other hand, if the correction was not justified we might have introduced a spurious correlation.

Although the slope differs for the different scenarios plotted in Fig. 1, the linear correlation coefficient does not. The correlation coefficient, $r$, depends on the scatter, which is the same for all three scenarios. We have simulated a large number of data sets and computed the correlation coefficient before and after the correction. Before the correction we consider the correlation between $\Delta m_{\text {est }}$ and Hubble diagram residuals. After the correction the correlation between $\Delta m_{\mathrm{est}}$ and $\epsilon=\Delta m_{\text {true }}-\Delta m_{\mathrm{est}}$ is considered. For all three scenarios, the initial correlation coefficient is $\langle r\rangle \simeq 0.27$. The uncertainty in the correlation coefficient decreases as $\sigma_{r} \simeq 1.3 / \sqrt{N}$ as the number of SNe Ia increases. After applying the correction, we find $\left\langle r_{\text {corr }}\right\rangle$ to be approximately $-0.02,0.09$, and -0.10 for the no shift, under-, and overestimation scenario. For the under- and overestimation scenarios, where the magnifications are not correctly estimated, the correction results in weak spurious correlations. Whether $r$ and $r_{\text {corr }}$ are significantly different or not depends on the sample size. For the no shift scenario, the difference would be significant (above $3 \sigma$ ) for $N \gtrsim 200$. If the correction coefficient was taken into account, $\Delta m_{\mathrm{est}}$ and $\epsilon=\Delta m_{\text {true }}-\Delta m_{\mathrm{est}}$ would be completely uncorrelated since $B$ was fitted to the data, making this test inappropriate.

\section{Discussion and summary}

Future SN Ia data sets are anticipated to be large, which could justify flux averaging as a method to overcome gravitational lensing bias. However, such large homogeneous data sets will probably allow for a reduction of systematic uncertainties and the use of subsets of SNe Ia - or the discovery of new correlations with peak brightness - may lead to large reductions of the intrinsic brightness dispersion. Under such circumstances, gravitational lensing corrections may play an important role in precision cosmology.

In this paper we have investigated the possibility of performing corrections for gravitational lensing of individual SNe Ia that reduce the scatter with negligible bias. Since one of the largest uncertainties in the estimation of magnification factors is the relation between luminosity and mass of galaxy haloes in the foreground, we have studied three different scenarios: no shift in halo masses, underestimation by $50 \%$ for all halo masses, and overestimation by $50 \%$ for all halo masses. Our simulations show that for all three scenarios, the scatter due to gravitational lensing can be reduced by roughly a factor of 2 for an SNLS-like data set. Also, the correction will be useful even if we do not know the luminosity-to-mass relation for the lensing galaxies to better than $50 \%$. Any apprehension that gravitational lensing corrections would lead to bias thus appears to be unfounded. For simulated SNLS-like data sets we found $\left|\langle\epsilon\rangle / \sigma_{\Delta m_{\text {true }}}\right| \lesssim 0.25$. The bias $\langle\epsilon\rangle$ is also expected to vary with redshift, but at a much smaller level $\left(\left|\langle\epsilon\rangle / \sigma_{\Delta m_{\text {true }}}\right| \lesssim 4 \times 10^{-2}\right)$.

As expected, the no shift scenario decreases the scatter more than the under- and overestimation scenarios. However, a simple correction coefficient, $B$, parameterizing the slope in the magnification-residual diagram, can increase the performance of the corrections of the under- and overestimation scenarios to the same level as the no shift scenario. Including the $B$-coefficient in the correction can hence eliminate the effect of bias in halo masses.

Correcting for gravitational lensing could reduce the total scatter in the SN Ia magnitudes by $4 \%$ for an intrinsic dispersion of $\sigma_{\text {int }}=0.13 \mathrm{mag}$ and errors similar to the ones obtained for SNLS (Astier et al. 2006). For $\sigma_{\text {int }}=0.09 \mathrm{mag}$, which might not be unrealistic for future surveys, the total scatter could be reduced by $6 \%$. Using Fisher matrix analysis we find that the size of the error ellipses in the $\left(\Omega_{\mathrm{M}}, w\right)$-plane can be reduced by 4-8\% for realistic measurement errors and realistic values of the intrinsic brightness scatter.

Gravitational magnification of SNe Ia is rather independent of cosmological parameters. The cosmology dependence on the corrections should thus be rather small. However, when a more elaborate correction, such as Eq. (5), is employed which requires a fit to data, we have to be more careful. The residuals used depends on the cosmology and a proper correction must take this into account. One solution would be to iteratively compute the slope in the magnification-residual diagram and estimate the cosmological parameters. We note that the gravitational lensing magnification we consider here affects all points of the SN Ia light-curve by the same amount, making it possible to apply the corrections after the light-curve fit.

Corrections for gravitational lensing could also be important for other distance indicators. Gravitational waves emitted by chirping binary systems could - if detected - be used to obtain very accurate luminosity distances (Schutz 1986). Furthermore, if the redshift of the binary could be measured from an optical counterpart, these binary system could be standard sirens, the gravitational wave analogs of standard candles. These standard sirens are unaffected by most systematic uncertainties which plague standard candles and they might provide distances with a relative accuracy $\lesssim 1 \%$. Standard sirens and standard candles both undergo extra dispersion due to gravitational lensing. For standard sirens this effect is much more important than for standard candles, since the other uncertainties are so small. Gravitational lensing will thus degrade the power of chirping binary systems as distance indicators (Holz \& Hughes 2005). The potential improvement of correcting standard sirens for lensing 
was investigated in Jönsson et al. (2007). Corrections could restore some of their power. The results found here, that gravitational lensing corrections can be unbiased, thus could be of importance also for future precision gravitational wave cosmology.

Acknowledgements. The Dark Cosmology Centre is funded by the Danish National Research Foundation. E.M. and J.S. acknowledges financial support from the Swedish Research Council and from the Anna-Greta and Holger Crafoord fund. J.S. is a Royal Swedish Academy of Sciences Research Fellow supported by a grant from the Knut and Alice Wallenberg Foundation.

\section{References}

Astier, P., Guy, J., Regnault, N., et al. 2006, A\&A, 447, 31

Cooray, A., Huterer, D., \& Holz D. 2006, Phys. Rev. Lett., 96, 021301

Dahlén, T., Mobasher, B., Somerville, R. S., et al. 2005, ApJ, 631, 126
Dalal, N., Holz, D. E., Chen, X., \& Frieman, J. A. 2003, ApJ, 585, L11

Frieman, J. A., Basset, B., Becker, A., et al. 2008, AJ, 135, 338

Gunnarsson, C. 2004, JCAP, 03, 002

Gunnarsson, C., Dahlén, T., Goobar, A., Jönsson, J., \& Mörtsell, E. 2006, ApJ, 640,471

Holz, D., \& Hughes, S. 2005, ApJ, 629, 15

Holz, D. E., \& Linder, E. V. 2005, ApJ, 631, 678

Jönsson, J., Goobar, A., \& Mörtsell, E. 2007, ApJ, 658, 52

Jönsson, J., Kronborg, T., Mörtsell, E., \& Sollerman, J. 2008, A\&A, 487, 467

Leibundgut, B. 2008, General Relativity and Gravitation, 40, 221

Miknaitis, G, Pignata, G., Rest, A., et al. 2007, ApJ, 666, 674

Navarro, J. F., Frenk, C. S., \& White, S. D. M. 1997, ApJ, 490, 493

Sarkar, D., Amblard, A., Holz, D., \& Cooray, A. 2008, ApJ, 678, 1

Shutz, B. 1986, Nature, 323, 310

Wang, Y. 2000, ApJ, 536, 531

Wang, Y., \& Mukherjee, P. 2004, ApJ, 606, 654

Wood-Vasey, W. M., Miknaitis, G., Stubbs, C. W., et al. 2007, ApJ, 666, 694 